\title{
Aplicaciones del modelo On/Off al tráfico agregado en las redes de comunicaciones
}

\section{On-Off model applications to communication networks aggregated traffic}

\begin{abstract}
Andrés Parra León
Ingeniero electrónico y de telecomunicaciones, estudiante de la Maestría en Ciencias de la Información y las Comunicaciones de la Universidad Distrital Francisco José de Caldas. Bogotá, Colombia.aaparral@correo.udistrital.edu.co

\section{Elkin M. Piedrahita}

Ingeniero electrónico y de telecomunicaciones, estudiante de la Maestría en Ciencias de la Información y las Comunicaciones de la Universidad Distrital Francisco José de Caldas. Bogotá, Colombia.empiedrahitav@correo.udistrital.edu.co
\end{abstract}

\section{Octavio Salcedo}

Ingeniero en sistemas, magíster en Ciencias de la Información y las Comunicaciones, estudiante de Doctorado. Docente de la Universidad Distrital Francisco José de Caldas. Bogotá, Colombia. ojsalcedop@udistrital.edu.co

Clasificación del artículo: Revisión (Recreaciones)

Fecha de recepción: 20 de mayo de 2011

Fecha de aceptación: 29 de agosto de 2011

Palabras clave: Autosimilitud, autocorrelación, modelo de tráfico, movimiento Browniano fractal, parámetro de Hurst, tráfico On/Off.

Key words: Self-similarity, autocorrelation, traffic model, fractal Brownian motion, Hurst parameter, On/Off traffic.

\section{RESUMEN}

Los modelos que permiten recrear trazas de tráfico generadas en una red de comunicaciones, pueden utilizarse en predicciones y estimaciones de tráfico a fin de optimizar el uso de los recursos de la red; uno de estos es el modelo On/Off, el cual permite describir el comportamiento del tráfico agregado por una o más fuentes de información. La importancia de este artículo se centra en la incidencia que dicho modelo ha tenido al aplicarse en diversas tecnologías de comunicaciones, para 
esto se evalúan y contrastan diferentes tipos de documentos propuestos por varios autores. Con base en los resultados, podría decirse que los modelos On/Off aún siguen vigentes para el modelamiento de tráfico, y pueden ser aplicables en cualquier nivel del modelo ISO/OSI. Asimismo, los resultados observados indican que dependiendo del tipo de fuente On/Off (sencilla o multiplexada) los planteamientos varían en gran proporción, y que la complejidad matemática que se puede llegar a tener en los modelos con multiplexación de fuentes On/Off podría ser una limitante para tener en cuenta a la hora de su implementación.

\section{ABSTRACT}

The models to recreate traffic traces generated in a communications network can be used in predic- tions and estimates of traffic in order to optimize the use of network resources, one of these is the model On/Off, which allows describing the behavior of aggregate traffic by one or more sources of information. The importance of this article focuses on the impact that this model has been applied to various communications technologies, for this are evaluated and contrasted different types of documents proposed by several authors. Based on the results, arguably Models On/Off are still in place for traffic modeling, and can be applied at any level of ISO/OSI model. Also, the results observed indicate that depending on the type of power On/Off (single or multiple) the approaches vary greatly, and the mathematical complexity that can have models with source multiplexing On/Off could be a constraint to take into account in its implementation.

\section{INTRODUCCIÓN}

En la actualidad existen diferentes formas de modelar el tráfico en las redes de comunicaciones así como las fuentes que lo generan. Por ejemplo, con base en [1] los modelos de tráfico pueden ser clasificados como estacionarios y no estacionarios. Entre los modelos de tráfico estacionarios se encuentran los dependientes de rango corto y de rango largo. En los dependientes de rango corto se incluyen los procesos de Markov y los modelos de regresión; por su parte, los dependientes de rango largo involucran técnicas de modelado de tráfico como F-ARIMA, Movimiento Browniano Fractal y multiplexación de fuentes $\mathrm{On} / \mathrm{Off}$.

Se introduce así una clasificación de los modelos de tráfico, ya que es un punto de partida para localizar la aplicación de los modelos On/Off que se tratarán en el artículo; para lo cual se encontró que al modelar una fuente sencilla de tráfico
On/Off, esta presenta un comportamiento de dependencia de rango corto; pero si se realiza un proceso de multiplexación estadística de fuentes On/Off, el comportamiento de dicho proceso presenta una dependencia de rango largo, al cumplir con ciertas características que se describirán en la sección 2 (Modelos de fuentes On/Off). En la Fig. 1, se realiza un esquema de clasificación de los modelos de tráfico, indicando la incidencia de los modelos de fuentes On/Off.

Debido a que el modelo de fuentes On/Off, también conocido como Packet Train Source Model, puede tener una dependencia de rango corto o largo según sea la implementación de una fuente sencilla o de una multiplexación estadística de fuentes respectivamente; es preciso aclarar, que dicha dependencia tiene una relación directa con la correlación de la señal a través del tiempo. Por tanto la correlación, el coeficiente de correlación, la covariancia y el parámetro de Hurst son algunos de los fundamentos que se tienen para 


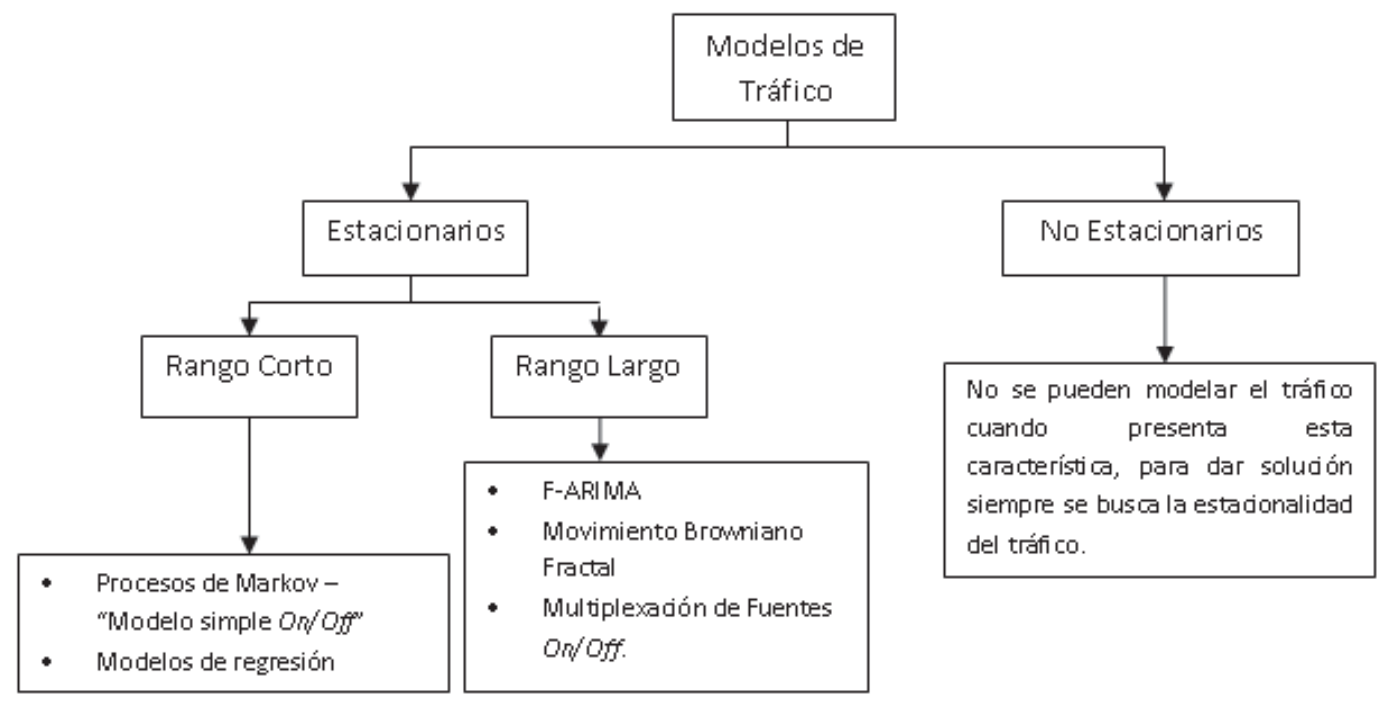

Fig. 1. Clasificación de modelos de tráfico.

ser críticos al momento de seleccionar el modelo que se pretende utilizar en un posible diseño.

Una vez que se selecciona y diseña un modelo que describa aproximadamente el comportamiento de una muestra de tráfico, es posible determinar cuáles factores afectan su desempeño, con el fin de estimularlos de manera tal que se puedan optimizar los procesos de transmisión y recepción, e inclusive llegar a predecir el comportamiento del tráfico lo que permitiría realizar una administración más óptima de los recursos que componen la red.

En este artículo se explica brevemente el funcionamiento de los modelos On/Off y se citan diferentes aplicaciones que se han hecho con este modelo en las redes de comunicaciones con el fin de alcanzar un estado del arte. Para esto, en la sección 2 se realiza una explicación de los modelos de fuentes $O n / O f f$ y se indican los tipos de fuentes que se han implementado. La sección 3 describe una serie de aplicaciones de los modelos $\mathrm{On} /$ Off propuestas por diferentes autores en área de las comunicaciones. En la sección 4 se presentan las discusiones de la información encontrada y se efectúa una clasificación de los documentos revisados. Finalmente, en la sección 5 se ofrecen las conclusiones que se obtuvieron acerca del estado del arte en la aplicación de modelos On/Off para el modelamiento de tráfico.

\section{MODELOS DE FUENTES ON/OFF}

Una de las formas más usadas para modelar el tráfico de una red, especialmente en el tráfico de voz, es mediante el uso de una fuente sencilla $O n / O f f$ [1]. Este modelo, se plantea en la década de los ochenta, pero también se extendió el uso de estas fuentes para modelar todo tipo de tráfico en Internet mediante la multiplexación de varias fuentes On/Off [2], lo que genera una mejora sustancial de este modelo, el cual retomó fuerza para la década de los noventa y, posteriormente, comenzó a dejar de utilizarse a principios del 2000 debido a que el modelado de grandes trazas de tráfico resulta difícil de trabajar en ecuaciones complejas con matemáticas, razón por la cual se deja esta tarea al Software. Aún así, se han encontrado documentos de los últimos cuatro años a la fecha, 
en donde se implementa el uso de este modelo de tráfico en tecnologías de red actuales o legadas, los cuales se describirán más adelante en la sección 3 (Aplicaciones de modelos On/Off).

El modelado de tráfico, se genera debido a que las redes de comunicaciones han presentado una tendencia a través del tiempo, la cual se centra en ofrecer a los usuarios diferentes tipos de servicios multimediales [3] que convergen en la red de un SP (Service Provider) independientemente de la tecnología de red que el proveedor implemente [4]. Como resultado, se presentan diversas trazas de tráfico propagadas hacia los usuarios, las cuales pueden ser representadas mediante series temporales para modelarlas en busca de pronosticar su conducta y mejorar el desempeño de la red a fin de ofrecer servicios con calidad.

Por ejemplo, para modelar una traza de tráfico mediante técnicas $O n / O f f$ se puede tomar la traza y representarla mediante una serie temporal basada en la metodología de Box-Jenkins. El desarrollo estadístico que se debe realizar con la serie temporal debe partir de un proceso estocástico estacionario [5][6]; es decir, mediante un proceso que cumpla con:

- Media constante

$$
E\left(Y_{t}\right)=\mu
$$

- Varianza constante

$$
\operatorname{Var}\left(Y_{t}\right)=E\left(Y_{t}-\mu\right)^{2}=\sigma^{2}
$$

- Correlación entre dos observaciones distintas iguales a la de otras dos observaciones cualquiera separadas por la misma distancia (mismo número de períodos).

$$
\operatorname{Cov}\left(Y_{t} ; Y_{t-j}\right)=\operatorname{Cov}\left(Y_{t+m} ; Y_{t+m-j}\right)
$$

Teniendo estas características, es posible continuar con el modelo basado en técnicas On/Off.

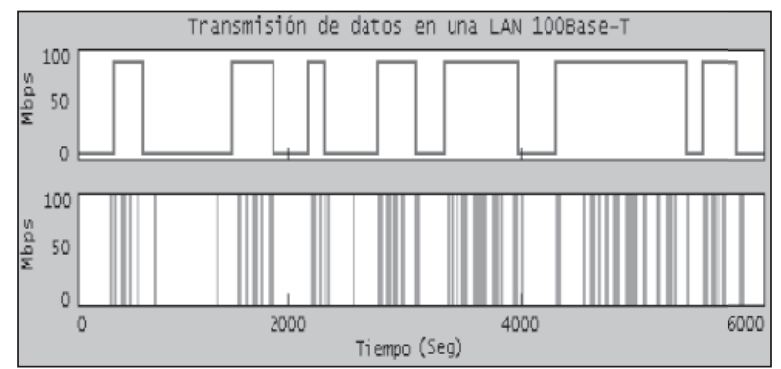

Fig. 2. Transmisión de datos por ráfagas en una red LAN 100Base-T con periodos ociosos (abajo), versus correspondencia del tráfico mediante modelo On/Off (arriba).

\subsection{Fuente sencilla On/Off}

Con base en los procesos estocásticos estacionarios y según sea la naturaleza de cada servicio brindado por el proveedor, es posible modelar las características del servicio como una fuente independiente que ofrece el flujo de datos en un estado activo (On) donde se genera tráfico a una tasa constante [7] y se alterna con un estado inactivo o de silencio (Off) en donde no se genera flujo de datos, de esta manera se constituyen intervalos de tiempo en los que se genera o no tráfico, los cuales son ilustrados en la Fig. 2.

Donde:

$\mathbf{X}$ es el tiempo dado en segundos.

Y es la transmisión dada en $\mathrm{Mb}$.

Por tanto, el comportamiento que describe el modelo On/Off para una fuente sencilla, presenta la transferencia de datos a una tasa de envió que es estipulada por las características de la fuente; pero que en el momento que se realiza la conmutación de estados, ya no se permite ningún tipo de transferencia de datos [8]. A su vez, los periodos On/Off se alternan y son I.I.D (Independientes e Idénticamente Distribuidos) [2], así como se observa en la Fig. 3.

Los intervalos de tiempo pueden tener una duración que responde a un comportamiento de dis- 


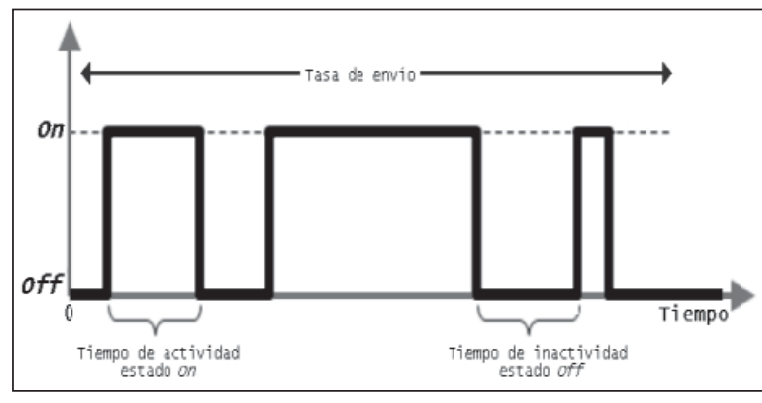

Fig. 3. Tiempos de estado On y Off con I.I.D en una fuente sencilla.

tribución exponencial [9][10], con media $\mu_{O n} \mathrm{y}$ $\mu_{\text {Off }}$, en donde la probabilidad de que el proceso se encuentre en el estado On está dada por:

$$
P_{O n}=\frac{\mu_{O n}}{\mu_{O n}+\mu_{O f f}} P_{O n}=\frac{\mu_{O n}}{\mu_{O n}+\mu_{O f f}}
$$

En estos procesos, es posible capturar la dependencia de rango corto mediante el cálculo de la autocovarianza [11]:

$$
\gamma(k) \approx A \exp (-\alpha|k|), \quad \forall \alpha>0
$$

El comportamiento de estos modelos conocidos como Poisson, ha sido complementado al agregar el concepto de estados que ofrecen las cadenas de Markov; dando lugar al Proceso de Poisson Modulado por Markov(MMPP, Markov Modulated Poisson Process) [12] en el que se combina el agregado de procesos estadísticamente independientes de Poisson (Teorema de Palm) con la conmutación Markoviana que involucra la propiedad de Markov, en la cual se implica que el futuro depende del estado actual y no de los estados previos ni del tiempo en cada estado.

De esta manera se obtienen ventajas entre las que se encuentran la capacidad de ser aplicados a distintos tipos de tráfico [13 - 15] y la sencillez en la implementación por una dependencia de rango corto, lo cual conlleva a tener una alta Parsimonia que está directamente relacionada con la cantidad de estados que tenga la cadena de Markov. Es decir, que a medida de que aumente la cantidad de

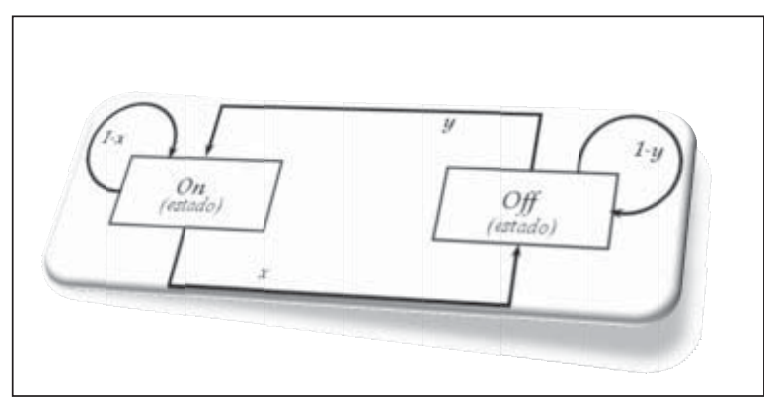

Fig. 4. Modelo de Fuente Individual On/Off.

estados se agregará complejidad significativa que disminuirá la Parsimonia.

Se debe aclarar que estos modelos tienen una desventaja notoria, ya que al ser dependientes de rango corto no cumplen con una descripción muy aproximada en el comportamiento del tráfico; lo que llevó a realizar investigaciones [2] en las que se demostró que en diversas trazas de tráfico, como la cantidad de datos que arriban en un instante de tiempo no dependen únicamente de su estado anterior, sino que también dependen de varios de los estados inmediatamente anteriores, por lo que se percibió una autocorrelación considerable.

Para la representación sencilla de una fuente $\mathrm{On} /$ Off, se utiliza el modelo de CTMC (ContinuousTime Markov Process) ilustrado en la Fig. 4.

Dónde:

$\boldsymbol{x}$. es la probabilidad de transitar del estado $O n$ al estado Off.

$\boldsymbol{y}$. es la probabilidad de transitar del estado $O f f$ al estado $\mathrm{On}$.

\subsection{Multiplexación de fuentes On/Off}

La técnica de modelado de fuentes $O n / O f f$ brinda otro enfoque interesante, el cual consiste en la multiplexación de varias fuentes que describen 
procesos On/Off con distribuciones de probabilidad de variables $[16,17]$. Esta técnica se basa en el comportamiento de una variable aleatoria () [11] que tiene una distribución de cola pesada, si se presenta qué:

$$
P[V A>x] \approx c x^{-\alpha}, \quad x \rightarrow \infty
$$

Dónde:

$V A$, es la variable aleatoria.

$c$, es una constante positiva.

$\alpha$, es el parámetro de forma o índice de la cola de la distribución para valores de .

Al analizar la respuesta de la ecuación (6) es posible observar que si la cola tiene una distribución complementaria exponencial, esta decaerá hiperbólicamente al cumplir con la condición de , lo que se hace notorio al contrastar este comportamiento por ejemplo con una distribución complementaria de Pareto, la cual decaerá describiendo una pendiente decreciente, ilustrado en la Fig. 5; característica que se da en gran medida por el efecto "Noah" (síndrome de varianza infinita) presentado en cada una de las fuentes $\mathrm{On} /$ Off que participan del proceso de multiplexación estadística.

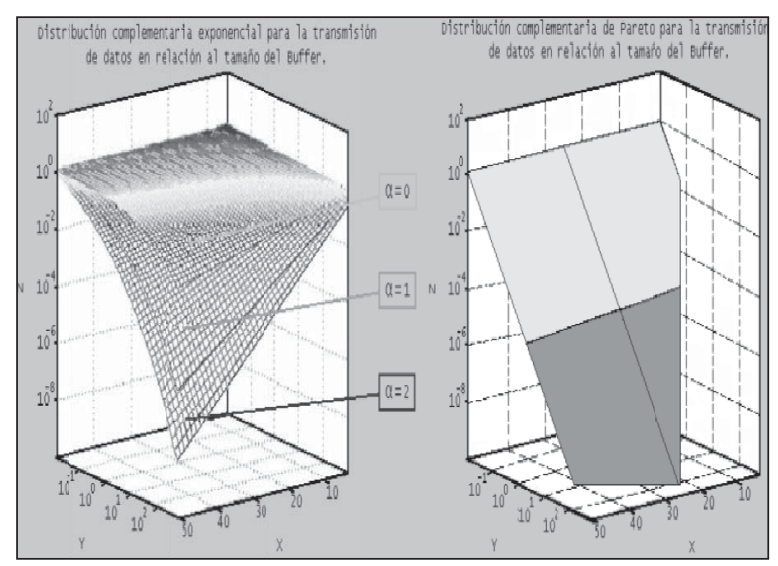

Fig. 5. Contraste de la transmisión de datos modelada mediante una variable aleatoria con distribución Exponencial (izquierda) y de Pareto (derecha) en relación al tamaño del Buffer. Fuente [18].
Dónde:

$\mathbf{X}$ representa el tamaño del Buffer.

Y está dado en segundos.

$\mathbf{Z}$ está dado en MB.

Si la duración en cada uno de los estados del modelo On/Off es exponencial y se mantiene la característica I.I.D (Independientes e Idénticamente Distribuidos) con un comportamiento de cola pesada, se podría describir su funcionamiento mediante una cadena de Markov[19] y de esta manera modelar tráfico con dependencia de rango largo, tal como se realiza en [20]; donde se sugieren los modelos de la Fig. 6 con secuencias On/ Off para el tráfico de datos en redes de comunicaciones como Internet:

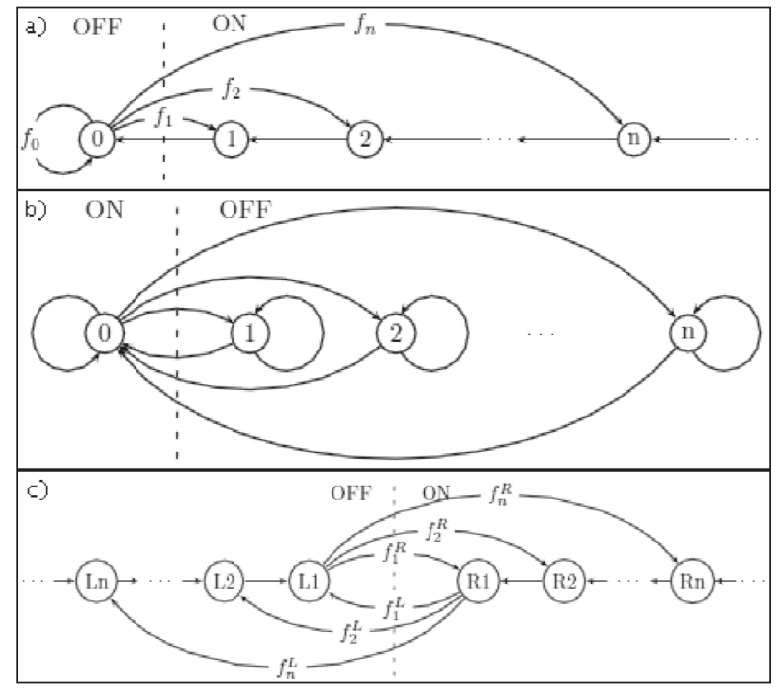

Fig. 6. Modelos para el comportamiento del tráfico de datos en Internet mediante procesos de Markov. a) Modelo que genera LRD (Long RangeDependence) para distintos valores de . b) Modelo PSST (Pseudo-Self-Similar Traffic) para $\mathrm{n}$ estados. c) Modelo Arrowsmith/Barenco hasta los estados Ln y Rn. Fuente [20].

Estos potenciales modelos son presentados para describir el tráfico de datos que genera una de las fuentes que son multiplexadas mediante procesos dependientes de rango largo; es decir, ofreciendo correlaciones a grandes intervalos de tiempo. 


\section{re-creaciones}

Las muestras de tráfico resultantes de este proceso tienen autocorrelación y describen una dependencia de rango largo, por lo que se obtiene autosimilitud o tráfico fractal, el cual puede ser modelado como un Movimiento Browniano Fractal [21,22]. Un ejemplo de esta técnica de modelado se muestra a continuación en la Fig. 7:

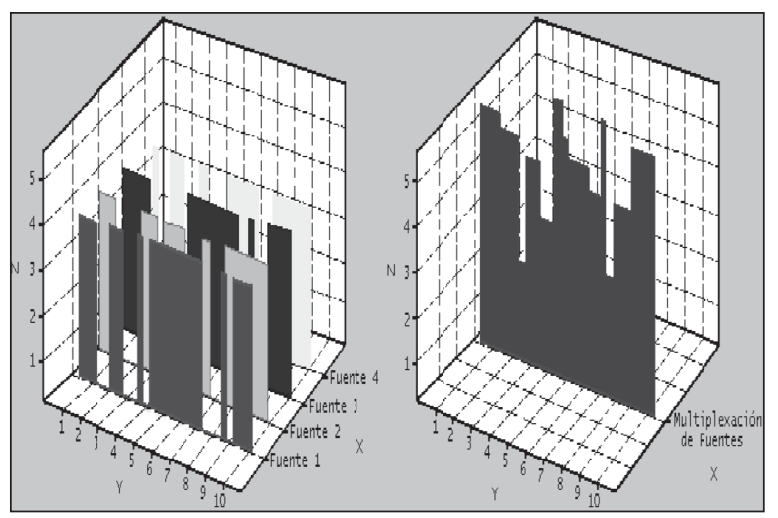

Fig. 7. Multiplexación de fuentes On/Off.

Dónde:

$\mathbf{X}$ (a la izquierda) es la cantidad de fuentes involucradas en el proceso, (a la derecha) es el tráfico resultante del proceso de multiplexación.

$\mathbf{Y}$ es el tiempo dado en minutos.

$\mathbf{Z}$ es la cantidad de datos dados en MB.

Las fuentes $O n / O f f$ envían tráfico durante su estado activo, el cual es representado por la variable aleatoria [23] que describe un comportamiento de cola pesada [24]e involucra parámetros específicos de con los cuales se demuestra en [25] que el resultado de la multiplexación estadística de fuentes dado por presenta una tendencia hacia un Movimiento Browniano Fractal [26] que está directamente relacionado con la distribución probabilística de los estados activos e inactivos de la fuente $O n / O f f$. Por consiguiente:

$$
Y_{N}(t) \approx \frac{E\left[\tau_{O n}\right]}{E\left[\tau_{O n}\right]+E\left[\tau_{O f f}\right]} N t+c \sqrt{N} B_{H}(t)
$$

Dónde:

$N$, es la cantidad de fuentes que participan del proceso de multiplexación estadística.

$B_{H}(t)$, es el Movimiento Browniano Fractal que presenta una varianza de 1 y un parámetro de Hurst dado por

$H=(3-\alpha) / 2$

$c$, es una constante positiva que depende únicamente de las distribuciones de $\tau_{O n}$ y $\tau_{O f f}$.

De esta forma, se ofrece una manera de modelar tráfico con características autosimilares y de cola pesada, mediante técnicas basadas en modelos On/Off; ya que si se analiza una captura de tráfico realizada en una red de comunicaciones es posible abstraer que "el tráfico es una serie de tiempo" [27] la cual puede ser representada en un plano de magnitud versus tiempo en busca de diseñar o encontrar un modelo matemático que describa su comportamiento, con el que se podría realizar un pronóstico [28] aproximado de la aleatoriedad que tienen sus cambios.

Este análisis de tráfico se utiliza actualmente para implementar aplicaciones como las rutinas de mitigación y corrección preventiva, a fin de ofrecer calidad en el servicio ( $Q o S$, Quality of Service) $[3,29,30]$ evitando que sucedan inconvenientes como el sobre-flujo (Overflow) de información en la red, que ocasiona grandes colas de paquetes y la excesiva carga en la CPU (Central Processing Unit), que en el peor de los casos conlleva a la pérdida de la información por la posible saturación de los Buffers en los dispositivos de la red.

Existe una variedad de formas para modelar el tráfico, entre las que actualmente se destacan los modelos basados en auto-similitud o fractalidad del tráfico, ya que proporcionan una herramienta importante para el análisis y diseño de los modelos de tráfico, debido a que mediante procesos de 


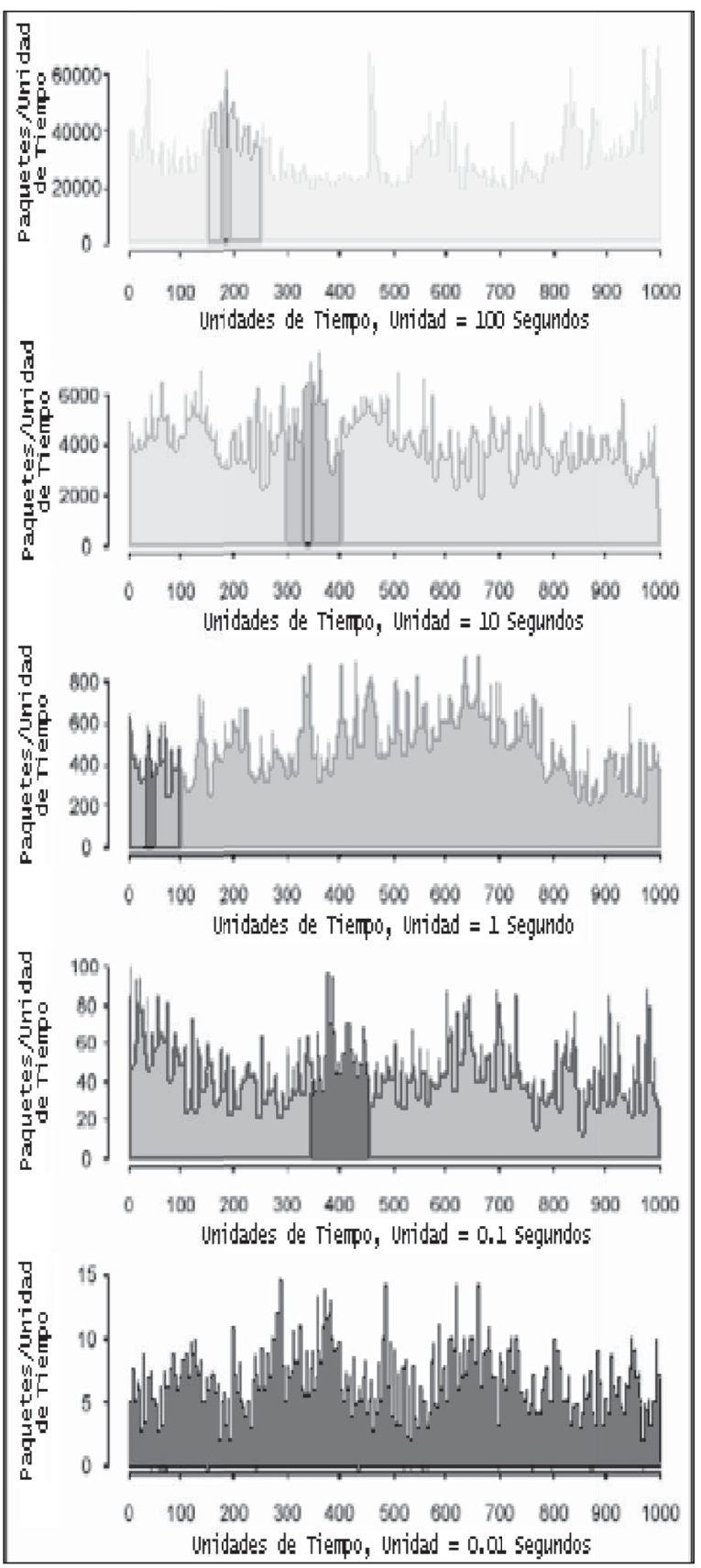

Fig. 8. Paquetes por unidad de tiempo en una red Ethernet vista en 5 escalas diferentes $(100,10$, $1,0,1$ y 0,01 segundos). Fuente [31].

"prueba visual de autosimilitud" es posible observar que el tráfico se comporta de la misma manera a diferentes escalas de tiempo, tal como se ilustra en la Fig. 8.
De esta forma, se encuentra que al modelar el tráfico de un segmento de tiempo es posible predecir su comportamiento en el siguiente instante (hora, día, mes e incluso año), lo cual estará directamente relacionado con qué tan grande es la dependencia de rango largo que ofrece el modelo frente al patrón de tráfico analizado.

Dónde:

$\mathbf{X}$ es en tiempo dado en segundos a diferentes escalas.

Y es la cantidad de paquetes por unidad de tiempo.

Se debe indicar, que el tiempo de permanencia en cada estado On/Off para el tráfico convencional, estaba caracterizado por distribuciones de colas ligeras que decaían de forma exponencial [32]. Aunque en [2] sí se llegó a considerar que este tipo de tráfico convencional como por ejemplo el de Ethernet por sus particularidades presenta tiempos de permanencia altos en cada estado, a lo que se le conoce como colas pesadas. De modo que la varianza en los tiempos de permanencia en cada estado podría llegar a ser infinita. Por tanto, el tráfico generado por las fuentes en el modelo On/Off se caracteriza por tener periodos inactivos variables y una transmisión por ráfagas de longitud variable en los estados activos; con la limitante de que la transferencia de datos mediante ráfagas no puede exceder el Buffer del destino, porque de ser así se comenzaría a perder información.

\section{APLICACIONES DE MODELOS ON/ OFF}

Al realizar una revisión detallada de las aplicaciones más relevantes de los modelos On/Off, para llegar y enfatizar en los trabajos desarrollados en los últimos 4 años, se encontró:

En [7] se realiza la multiplexación estadística de fuentes On/Off en redes ATM (Asynchronous 


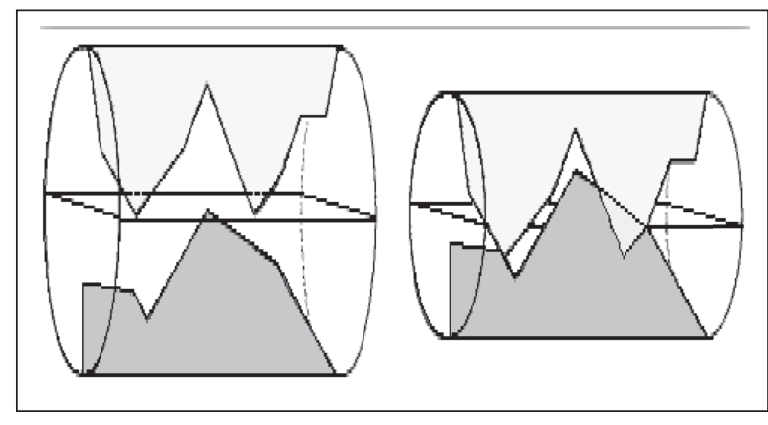

Fig. 9. A la izquierda: Redes tradicionales, uso ineficiente de los recursos en la transmisión. A la derecha: Multiplexación estadística de fuentes en ATM sobre un enlace de baja capacidad, existe la probabilidad de que se produzcan conflictos pero se ocupa menos ancho de banda. Fuente [7].

Transfer Mode) en busca de darle un mejor uso a los recursos de ancho de banda disponibles en la red impactando directamente en la eficiencia de los servicios que se prestan a través de ATM. Se realiza un contraste de la red ATM con multiplexación de fuentes en enlaces de baja capacidad frente a las redes tradicionales que reservan recursos de ancho de banda equivalentes al valor pico de la tasa de transferencia de cada servicio, y se evidencia un uso ineficiente del ancho de banda por parte de las redes tradicionales. Se especifica que la sanción por utilizar un método estadístico, es que existe la probabilidad de que las transferencias más altas de dos servicios coincidan en un instante de tiempo y se pierda información por sobre-flujo impactando en la QoS, tal como se ilustra en la Fig. 9.

En dicho artículo se expone que en busca de minimizar los efectos de sobre-flujo en donde se puede perder información y en pro de mantener la QoS es posible implementar Controles de Admisión de Conexión (CAC) y mecanismos de control de congestión, lo cual se fundamenta en el modelado de fuentes y de tráfico (May. 1998).

En [33] se realiza un análisis del tráfico de datos en redes de acceso ADSL (Asymmetric Digital
Subscriber Line), como característica importante observan que las distribuciones de tráfico pueden ser representadas por distribuciones de cola pesada con caída hiperbólica; es decir, que tienen dependencia de rango largo y se puede modelar con técnicas $O n / O f f$.

Asimismo, se describe que existen otras distribuciones que pueden ser analizadas e incluidas dentro de otros modelos de tráfico para caracterizar la variabilidad del tráfico como la de Pareto, Weibull y Longnormal; de las cuales se abstrae la distribución más aproximada al tráfico de datos que se analiza en ADSL es la de Weibull. Se fundamentan los resultados mediante una comparación de los datos obtenidos en la simulación ilustrada en la Fig. 10.

Se concluye el artículo planteando que la red de acceso ADSL puede ser modelada mediante un sistema que se compone por una cola $\mathrm{G} / \mathrm{G} / 1$ la cual representa un dispositivo de red CPE (Customer-PremisesEquipment) y una cola M/G/1 que modela el DSLAM (Digital Subscriber Line Ac-

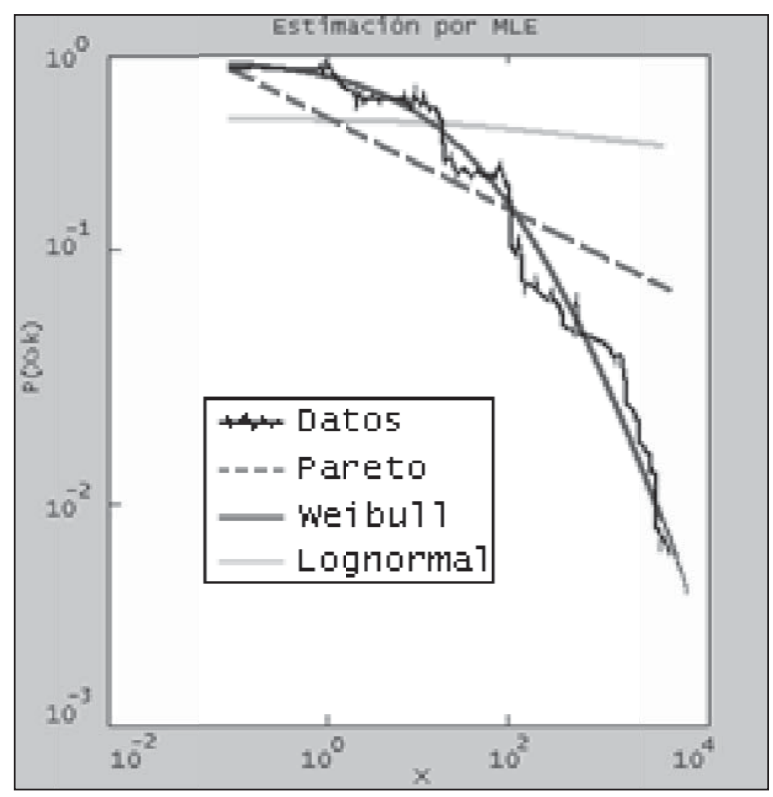

Fig. 10. Contraste de las distribuciones de cola pesada para tráfico de datos en redes de acceso ADSL. Fuente [33]. 
cess Multiplexer), en donde la distribución General (G) de mayor aproximación es en ambos casos la de Weibull (Sep. 2002).

En [34] se realiza un estudio de fractalidad en tráfico de VoIP. El análisis y los resultados indican que el proceso de Poisson presenta un buen comportamiento para modelar la llamada que ingresa mediante VoIP [35], pero se argumenta que los tiempos de llamada en espera muestran distribuciones de cola pesada en lugar de distribuciones exponenciales. Por tanto, en ese artículo se propone como valor agregado una distribución generalizada de Pareto para modelar los tiempos de espera entre las llamadas, dicha distribución puede ser utilizada para modelar de forma precisa los períodos activos y ociosos $(\mathrm{On} / \mathrm{Off})$ del tráficoVoIP que se presenta en cada llamada. El análisis concluye que el agregado de tráficoVoIP tiene características fractales y se sugiere el modelo fraccional de ruido Gaussiano para su comportamiento (2004).

En [36] se realiza una evaluación de la probabilidad de bloqueo de forma rápida para las conexiones de extremo a extremo (end-to-end) en una red de conmutación de ráfagas ópticas $(O B S, O p$ ticalBurstSwitching), con un modelo de tráfico de entrada On/Off no uniforme se logra encontrar la eficiencia del modelo como método para permitir el cálculo y el análisis óptimo en redes de gran tamaño, bajo tecnologías que basan la transmisión de información por medio óptico (Oct. 2006).

Muchos tipos de tráfico que se dan en una red de comunicaciones multi-medial tienen características modelables a través de procesos On/Off, pero debido a que las trazas de tráfico pueden tener comportamientos extraños como por ejemplo, que la estimación de parámetros de Hurst sean mayores que 1; genera que en [37] se analicen algunos de esos comportamientos atípicos. El citado documento, especifica que se debe prestar principal atención en dos aspectos fundamentales en el momento de utilizar el modelo On/Off para describir el tráfico de una red. El primero, consiste en que la parte inicial de la muestra del tráfico que se pretende analizar no es estacionaria y por tanto debe ser retirada. El segundo, parte de que la muestra del tráfico analizado podría llegar a ser muy grande, por lo que se pueden presentar tendencias que no son muy convenientes para establecer un modelo adecuado. Se llega a la conclusión, de que el análisis y la simulación de tráfico On/Off puede brindar resultados satisfactorios en los que los modelos describen de manera aproximada el comportamiento de una traza de tráfico, si dicha traza cumple con un intervalo de muestreo no muy grande y si la parte inicial de la traza que no es estacionaria ha sido retirada (2008).

En [38] se ofrece un modelo de tráfico de voz On-Off-2On el cual es extrapolado a un medio de transmisión inalámbrico (Wireless) y podría incorporarse en el diseño genérico Cross-Layer. En conjunto, se propone un método analítico para evaluar el impacto de los parámetros del tráfico de voz sobre el rendimiento de la red de acceso inalámbrico. Se fundamenta el artículo, destacando como particularidad del tráfico de voz, que el período de ráfagas de paquetes en los momentos de actividad $O n$ tiende a ser distribuido de forma independiente, en donde las trazas de tráfico que se presentan en dichas ráfagas tienden a ser fuertemente correlacionadas durante los tiempos de conversación; se especifica que esta característica es importante en las WLAN/WMAN para la planificación de recursos, ya que tanto la transmisión como la recepción en un diálogo de voz se realiza a través del mismo medio común. (2008).

En [39] se hace una regresión no paramétrica para datos funcionales no estacionarios, el trabajo tuvo como propósito analizar la dependencia y no estacionalidad del tráfico en el enlace de una red. El modelo On/Off tuvo su aplicación para simular el tráfico sobre el enlace, donde los tiempos de $O n$ y $O f f$ se manejaron como variables exponenciales 
independientes en busca de simular el comportamiento no estacionario del tráfico. Por medio de una variable se seleccionó periódicamente entre el régimen de poco tráfico y el de mucho tráfico. En cada proceso On/Off que se realizó, se encontró una función de distribución empírica del tiempo entre los paquetes enviados y el retardo promedio, con lo que se concluyó que los datos presentaron periodicidad al alternarse los regímenes de mucho y poco tráfico, lo cual indica que la distribución empírica responde al modelo planteado en el documento. (Sep. 2008).

En [40] se consideró que un modelo On/Off es capaz de capturar la naturaleza por ráfagas de una fuente de tráfico generada por una red de sensores. Dicha red, tiene como fin monitorear objetos en movimiento de forma colaborativa; así, cuando un objeto entra en el área segura cualquier sensor con la habilidad de monitorear indicará que el objetivo se encuentra en el área, de este modo, el sensor relacionado generará ráfagas de tráfico que serán capturadas con el modelo On/Off. Para conseguir esto con el modelo On/Off, se relaciona el estado $O n$ a un intervalo de tiempo en el que un objeto aparece dentro del rango de cobertura del sensor y el estado Off representa un intervalo de tiempo donde el objeto se encuentra fuera de la zona de cobertura. El autor realiza una observación indicando que los periodos de distribución On y Off siguen una distribución generalizada de Pareto independiente de la ubicación del sensor que se considera como un receptor y del tiempo que este permanezca en estado On. (Oct. 2008).

En [41] se brinda un modelo escalable y aproximado que permite el análisis de la pérdida de paquetes de longitud variable que se presenta en Switches con Buffers finitos. El análisis se realiza con el modelo On/Off representando los estados de entrada y salida de información en Switches con Buffers de gran tamaño, a fin de contribuir con futuras ampliaciones en el tamaño del Switch, debido a que entre mayor tamaño su complejidad computacional aumenta por la relación directamente proporcional que se tiene con el número de puertos del Switch (2009).

En [42] se usan los modelos de Poisson, los modelos basados en secuencias de renovación agregadas y los modelos construidos a partir de fuentes $O n / O f f$ para analizar la conducta de cola pesada en la transmisión de tráfico de paquetes en los enlaces de comunicación de alta velocidad. En cuanto a la respuesta obtenida por las fuentes On/Off, se concluye que si las fuentes se conectan a una tasa vertiginosa a través del tiempo, las fluctuaciones estadísticas acumuladas generadas serán el Movimiento Browniano Fractal. Sin embargo, si la velocidad de conexión es lenta las fluctuaciones del tráfico son descritas por un proceso estable de Lévy [43], mientras que el límite de las fluctuaciones para la escala intermedia (teorema del límite central) está dado por un Movimiento Fractal de Poisson (Apr. 2010).

En [44] se plantea una técnica para construir un modelo DBMAP (Discrete-time BatchMarkovianArrivalProcess) [45], el cual se apoya en un modelo IPP (InterruptedPoissonProcess) [46] basado en $O n / O f f$ a fin de abordar los problemas de ingeniería de tráfico dados en las comunicaciones inalámbricas (Wireless). Se presenta, que el modelo derivado tiene alta parsimonia y que permite aplicar los métodos de la matriz de análisis en la evaluación del desempeño de las tecnologías de redes inalámbricas emergentes (2010).

En [47] se proponen modelos basados en cadenas de Markov en busca de describir los estados en los que se ocupan o no las longitudes de onda disponibles de una red de acceso óptico. Conjuntamente se desarrollan modelos sobre redes ópticas hibridas TDM-WDM (Time Division Multiplexing - Wavelength Division Multiplexing) para calcular la probabilidad de pérdida (generada por la falta de asignación de longitudes de onda) y para la probabilidad de bloqueo de llamadas (generada 
por la capacidad de ancho de banda restringido que tiene una longitud de onda). Se fundamentan los modelos debido a que la red de acceso óptico tiene la capacidad de ofrecer servicios mediante ráfagas $O n / O f f$ (Jul. 2010).

\section{DISCUSIONES}

Adicionalmente, con base en las aplicaciones planteadas a través de los artículos y de los trabajos encontrados del modelo On/Off a la fecha; se presenta una posible clasificación mediante la Fig. 11, en donde se relacionan las aplicaciones del modelo On/Off a diferentes tipos de tráfico y tecnologías de red:

En la Fig. 11 se ilustra que la mayor tendencia se centra en el uso de las características de dos estados (On/Off) como técnica de modelado para diferentes procesos que se realizan en las redes de telecomunicaciones, así como en el tráfico que las redes generan $[48,49,51]$. La aplicación más relevante dada en el modelado es seguida de una gama de recomendaciones ITU-T (International Telecommunication Union - Standardization Sector) que se dan a través de estándares para el modelado, la medición y la previsión de tráfico.

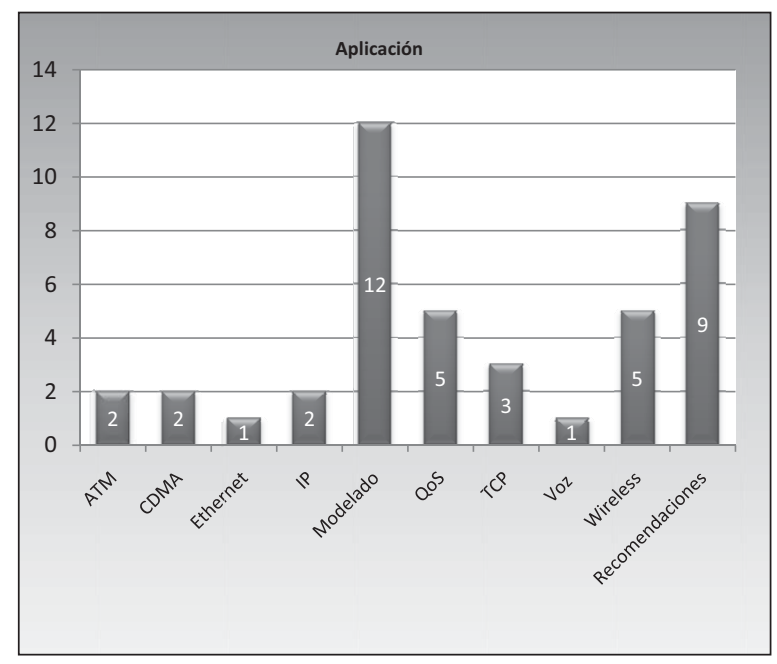

Fig. 11. Clasificación de los artículos por tipo de aplicación.
Sin embargo, se encontró que la utilización del modelo On/Off también es implementada a lo largo del modelo de referencia ISO/OSI (International Standard Organization / Open System Interconnection) debido a que es posible interpolar las características descritas con este modelo en varios tipos de tráfico y tecnologías de red que se encuentran en diversos niveles o capas de OSI, tal como se describirá a continuación:

- A nivel de capa 1, se presenta el uso del modelo On/Off, tanto en el tráfico como en tecnologías de acceso inalámbrico (Wireless) [38,44,52], Ad-Hoc [53], en CDMA (Code Division Multiple Access) [54,55] y redes de transmisión óptica como WDM [36].

- En capa 2, la implementación del modelo On/ Off es enfocada a modelar el tráfico agregado a fin de optimizar los recursos de las redes con enlace de red ADSL [33], Ethernet y ATM $[7,56,57]$, lo que permite pensar que se podría utilizar este modelo en otras tecnologías de capa 2 como por ejemplo FrameRelay, ya que cumple con características similares a las que se encuentran en ATM. Aún así, también se encontró que mediante el modelo On/Off se puede analizar la viabilidad para ampliar los Buffers en Switches de gran capacidad [41], eliminando parte de la complejidad computacional que esto involucra.

- El tráfico IP (Internet Protocol) describe comportamientos auto-similares así como conductas activas y ociosas, por tanto en capa 3 es posible encontrar artículos que utilizan el modelo On/Off para capturar la estructura del tráfico IP tratando de describir su comportamiento $[15,58]$. Sumado a esto, por la particularidad del tráfico de voz al tener períodos con transmisión de ráfagas de información en los momentos de actividad y otros períodos de silencio, se obtienen implementaciones del modelo On/Off para tratar de representar 
la conducta del tráfico de VoIP y analizar la fractalidad que este pueda llegar a tener [34].

- En cuanto a la capa 4, la forma en la que se comporta el tráfico de entrada en protocolos como TCP (Transmission Control Protocol) según sean sus características puede ser modelado mediante técnicas $O n / O f f[59,60]$.

- Por otro lado, la calidad de servicio (QoS) se puede presentar a diferentes capas de OSI, ya que al aplicar el modelo On/Off en diversos tipos de tráfico se impacta [61] directamente en los tiempos de transmisión y recepción.
Esto permite reducir el encolamiento [62] que se da en los Buffers de los dispositivos que componen la red, ocupando menor cantidad de recursos físicos que se pueden reservar para ocasiones de mayor demanda [63], lo que ayuda a tener un mejor procesamiento, al igual que un mejor desempeño de la red.

En la tabla 1, se presenta un contraste de los documentos revisados para el análisis del estado del arte de la aplicación de los modelos On/Off, organizando los resultados de las investigaciones de acuerdo con el tipo de documento, título, aplicación y año de publicación. Esta tipificación

Tabla 1. Documentos de aplicaciones de fuentes On/Off.

\begin{tabular}{|c|c|c|c|}
\hline Tipo de Documento & Título & Aplicación & Año \\
\hline Estándar & ITU-T E.507 & Recomendaciones & 1988 \\
\hline Estándar & ITU -T E.711 & Recomendaciones & 1992 \\
\hline Estándar & ITU -T E.712 & Recomendaciones & 1992 \\
\hline Estándar & ITU -T E.713 & Recomendaciones & 1992 \\
\hline Estándar & ITU -T E.506 & Recomendaciones & 1992 \\
\hline Estándar & ITU -T E.508 & Recomendaciones & 1992 \\
\hline Estándar & ITU -T E.716 & Recomendaciones & 1996 \\
\hline Artículo en revista & $\begin{array}{l}\text { Characterization of ATM On-Off Traffic from Cell Traffic Measure- } \\
\text { ments }\end{array}$ & ATM & 1999 \\
\hline Artículo en revista & $\begin{array}{l}\text { Performance of a joint CDMA/PRMA Protocol with Heavy-Tailed On/ } \\
\text { Off Source }\end{array}$ & CDMA & 1999 \\
\hline Artículo en revista & $\begin{array}{l}\text { Capacity Estimation for an SIR-Based Power-Controlled CDMA Sys- } \\
\text { tem Supporting ON-OFF Traffic }\end{array}$ & CDMA & 2000 \\
\hline Artículo en revista & $\begin{array}{l}\text { A Frequency Domain Empirical Likelihood for Short and Long-Ran- } \\
\text { ge Dependence }\end{array}$ & Modelado & 2000 \\
\hline Artículo en revista & MMPP Models for Multimedia Traffic & Modelado & 2000 \\
\hline Estándar & ITU -T E.760 & Recomendaciones & 2000 \\
\hline Artículo en revista & Behavior of TCP-like Elastic Traffic at a Buffered Bottleneck Router & TCP & 2001 \\
\hline Artículo en revista & $\begin{array}{l}\text { Impact of Aggregated, Self-similar On/Off Traffic on Delay in Statio- } \\
\text { nary Queueing Models }\end{array}$ & Modelado & 2001 \\
\hline Artículo en revista & Designing Traffic Profiles for Bursty Internet Traffic & QoS & 2002 \\
\hline Artículo en conferencia & $\begin{array}{l}\text { A Performance Model and Analysis of Heterogeneous Traffic with } \\
\text { Heavy Tails }\end{array}$ & Modelado & 2003 \\
\hline Artículo en revista & Modeling TCP-Vegas under On/Off Traffic & TCP & 2003 \\
\hline
\end{tabular}




\begin{tabular}{|c|c|c|c|}
\hline Estándar & ITU -T E.490.1 & Recomendaciones & 2003 \\
\hline Sección libro & $\begin{array}{l}\text { Call-Burst Blocking Probabilities of On-Off Traffic Sources under } \\
\text { the Bandwidth Reservation Policy }\end{array}$ & QoS & 2004 \\
\hline Artículo en revista & Fractal Analysis and Modeling of VolP Traffic & Voz & 2004 \\
\hline Artículo en revista & Small Aggregations of On/Off Traffic Sources & Modelado & 2004 \\
\hline Artículo en revista & $\begin{array}{l}\text { State transition probability for the Markov Model dealing with On/Off } \\
\text { cooling schedule in dwellings }\end{array}$ & QoS & 2004 \\
\hline Artículo en conferencia & $\begin{array}{l}\text { Efficient simulation of a queueing system fed by general On/Off in- } \\
\text { puts }\end{array}$ & Ethernet & 2005 \\
\hline Artículo en revista & $\begin{array}{l}\text { Network and user driven alpha-beta On-Off Source Model for Net- } \\
\text { work Traffic }\end{array}$ & TCP & 2005 \\
\hline Tesis doctoral & $\begin{array}{l}\text { Traffic Balancing: A Method for Exploiting System Capacity in Wire- } \\
\text { less Ad Hoc Networks }\end{array}$ & Wireless & 2005 \\
\hline Artículo en revista & Bursty Traffic Simulation by On-Off Model & Modelado & 2006 \\
\hline Tesis de maestría & Estudio de la Fractalidad del Tráfico en Redes Ad-Hoc sobre WLAN & Wireless & 2006 \\
\hline Artículo en revista & $\begin{array}{l}\text { Experimental validation of the On-Off packet-level model for IP tra- } \\
\text { ffic }\end{array}$ & IP & 2006 \\
\hline Artículo en conferencia & On/Off Models to Capture IP Traffic Structure & IP & 2006 \\
\hline Artículo en conferencia & $\begin{array}{l}\text { Performance Evaluation of IEEE 802.11e based on On-Off Traffic } \\
\text { Mode }\end{array}$ & Wireless & 2007 \\
\hline Estándar & IEEE P802.19WirelessCoexistence & Wireless & 2008 \\
\hline Artículo en conferencia & $\begin{array}{l}\text { Source Traffic Modeling in Wireless Sensor Networks for Target } \\
\text { Tracking }\end{array}$ & Wireless & 2008 \\
\hline Tesis de maestría & Regresión no Paramétrica para Datos Funcionales no Estacionarios & Modelado & 2008 \\
\hline Tesis doctoral & $\begin{array}{l}\text { Performance Evaluation by Aggregation of On-Off Traffic in Proces- } \\
\text { sor Sharing and Related Systems }\end{array}$ & Modelado & 2008 \\
\hline Artículo en conferencia & $\begin{array}{l}\text { Remarks Regarding Queuing Model and Packet Loss Probability for } \\
\text { the Traffic with Self-Similar Characteristics }\end{array}$ & QoS & 2008 \\
\hline Sección libro & $\begin{array}{l}\text { Performance Analysis of Call Admission Control For Streaming Tra- } \\
\text { ffic With Activity Detection Function }\end{array}$ & ATM & 2009 \\
\hline Sección libro & $\begin{array}{l}\text { Scalable Model for Packet Loss Analysis of Load-Balancing Swit- } \\
\text { ches with Identical Input Processes }\end{array}$ & Modelado & 2009 \\
\hline Artículo en conferencia & Traffic Modeling for Agile All-Photonic Network Dimensioning & QoS & 2009 \\
\hline Artículo en conferencia & Long-Range Dependence of Financial Time Series Data & Modelado & 2009 \\
\hline Artículo en revista & The On-Off Network Traffic Model Under Intermediate Scaling & Modelado & 2010 \\
\hline Artículo en conferencia & Practical Traffic Generation Model for Wireless Networks & Modelado & 2010 \\
\hline
\end{tabular}

de los documentos encontrados permite hacer un estimativo de cuántos documentos fueron desarrollados con respecto a la temática en los últimos años y también permite apreciar en qué tipo de documentos se han hecho las mayores contribu- ciones del modelo On/Off las cuales se presentan en la Fig. 12.

En los documentos revisados y presentados en la tabla 1 se abstrae que el mayor tipo de informa- 


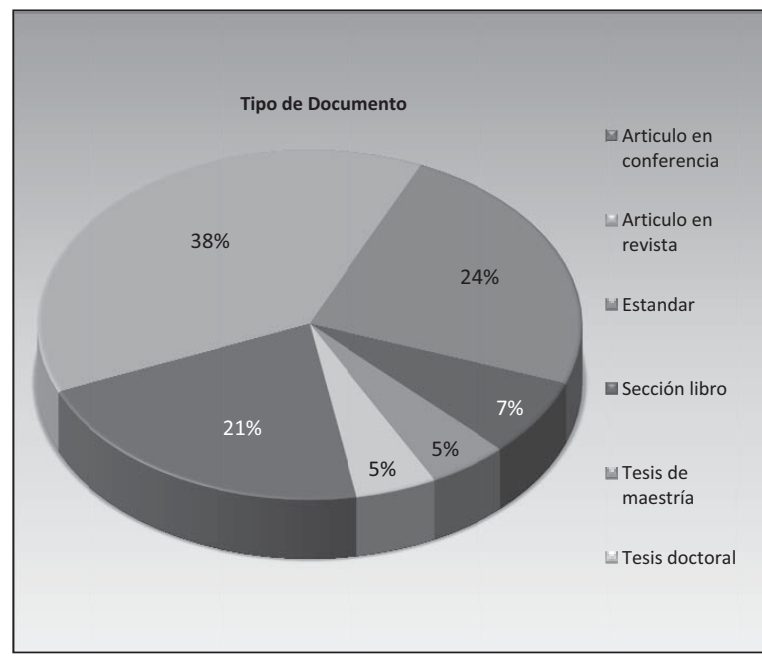

Fig. 12. Clasificación porcentual de los documentos con implementaciones On/Off revisados.

ción fue obtenido de los artículos publicados en revistas científicas, seguido de un interés que se destaca por parte de entidades reguladoras en el área de las telecomunicaciones como la ITU-T que ha orientado sus esfuerzos en pro de realizar estándares que se ofrecen para el modelado de tráfico. Por otro lado, los artículos provenientes de conferencias presentan el tercer porcentaje significativo, razón por la cual dichos artículos son una fuente importante de información para realizar trabajos futuros que involucren el modelado de tráfico mediante técnicas $O n / O f f$. Esta información se brinda de forma detallada en la Fig. 12, en la cual se ilustra que el modelo On/Off es un tema interesante para realizar investigación y desarrollo de Tesis a nivel de posgrado en busca de generar modelos que describan de forma aproximada el comportamiento de todo tipo de tráfico para realizar predicciones y contribuciones que permitan administrar de una mejor manera los recursos disponibles en las redes de comunicaciones.

Finalmente, en la Fig. 13 se ilustra la cantidad de documentos producidos en cada año. Los años 1996, 2002 y 2006 presentaron la menor cantidad de documentos relacionados con el modelo

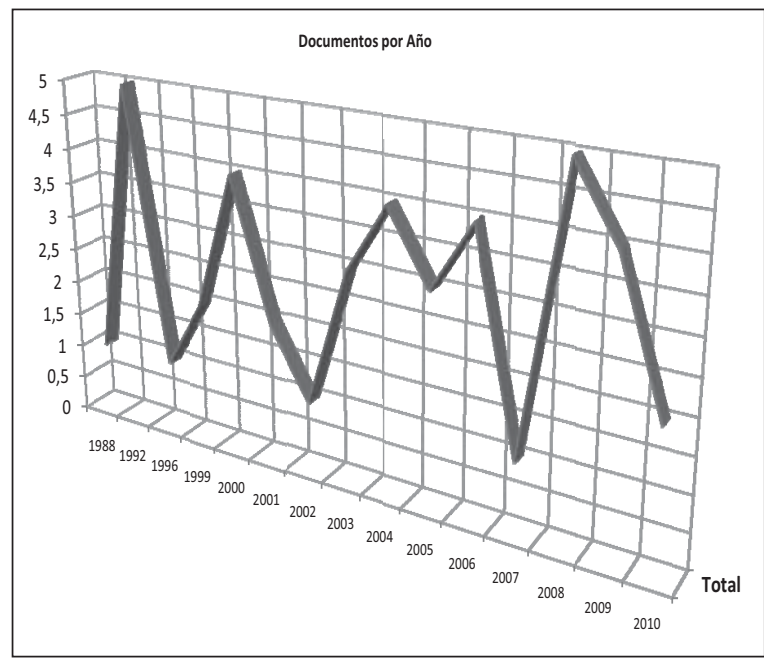

Fig. 13 Clasificación por año de publicación de los documentos revisados.

On/Off. Se podría pensar, que en dichos periodos de tiempo el modelado de tráfico basado en esta técnica iba a perder sus posibles aplicaciones en el ámbito tecnológico, pero al analizar la tendencia comprendida entre los años 2007 y 2008 se encontró una rápida recuperación en la aplicación del modelo On/Off en gran medida gracias a su Parsimonia, por tanto, el modelo On/Off sigue siendo utilizado para modelar tráfico. Sin embargo, como se mencionó con anterioridad, la complejidad matemática generada por las grandes trazas de tráfico hacen que el manejo del modelo On/Off decaiga en los últimos dos años, debido a que resulta menos complejo manipular los datos obtenidos por las trazas de tráfico ingresándolas en herramientas de Software, que ofrecen modelos computacionales eficientes y prácticos.

\section{CONCLUSIONES}

De acuerdo con la clasificación de modelos de tráfico, se encontró que el modelo de fuentes $\mathrm{On} / \mathrm{Off}$ puede ser un modelo estacionario de rango corto o largo, esto con base en la implementación de la fuente, bien sea de una forma sencilla o multiplexada; de igual manera, las aplicaciones que se 
dan en cada implementación varían dependiendo del tipo de tráfico que se desee modelar.

Los modelos On/Off tienen muchas ventajas con respecto a otros modelos de fuentes de tráfico, una de las más importantes es la parsimonia o sencillez de este modelo en el momento de implementarse, a pesar de esto el modelo no deja de ser complejo ante la presencia de un gran número de fuentes en una aplicación que requiera la multiplexación estadística de fuentes On/Off. En contraparte, como desventaja principal del modelo $O n / O f f$ se tiene que esta técnica de modelado es muy rígida, debido a que solo reconoce dos esta- dos, o se genera tráfico a una tasa constante o no se transmite nada, lo que no siempre se presenta en el comportamiento de un canal de comunicaciones.

En resumen, al estudiar el tráfico de una red es posible implementar y desarrollar aplicaciones que permitan ofrecer un uso más óptimo de los recursos que se tienen; así el modelo On/Off es una herramienta útil al momento de estimar y predecir aproximadamente la aleatoriedad del comportamiento de diferentes tipos de tráfico que presenten características autosimilares con I.I.D y una distribución de cola pesada.

\section{REFERENCIAS}

[1] A. Adas, "Traffic Models in Broadband Networks," IEEE Communications Magazine July. 1997.

[2] W. Willinger, "Self-Similarity in HighSpeed Packet Traffic: Analysis and Modeling of Ethernet Traffic Measurements," Statistical Science, vol. 10, pp. 67-85, Feb. 1995.

[3] M. Ajmone, G. Bianchi, M. Listanti and M. Meo, Quality of Service in Multiservice IP Networks. 2005.

[4] CA O'Flaherty, et ál, Transport Planning and Traffic Engineering. Butterworth: Elsevier, 2006.

[5] R. de Arce and I. Klein, Introducción a los modelos auto-regresivos con heterocedasticidad condicional (ARCH), 1998.

[6] Y. Jiang and Y. Liu, Stochastic Network Calculus, Springer-Verlag London Limited, 2008.
[7] D. Rincón, Introducción a los Modelos de tráfico para redes de Banda Ancha, Rama de estudiantes IEEE, 1998.

[8] F. Gebali, Analysis of Computer and Communication Networks, Springer Science + Business Media, LLC, 2008.

[9] P. Bravo, Modelado fractal de tráfico de la red de Ingeniería Telemática, Tesis de gradoUniversidad Carlos III de Madrid, 2004.

[10] G. Kesidis, An Introduction to Communication Network Analysis, New Jersey: Published by John Wiley and Sons, Inc. Hoboken 2007.

[11] M. Alzate y N. Peña, "Modelos de Tráfico en Análisis y Control de Redes de Comunicaciones", Revista Ingeniería, vol. 9, pp. 63-87, Universidad Distrital Francisco José de Caldas, 2004.

[12] S. Shah-Heydari and T. Le-Ngoc, "MMPP models for multimedia traffic," Telecom- 
munication Systems, vol. 15, pp. 273-293, Feb. 2000.

[13] K. Park and W. Willinger, Self-Similar Network Traffic and Performance Evaluation, New York: John Wiley and Sons, 2000.

[14] M. Alzate, "Simulation model for MPEGII Video Traffic". University of Maryland, ENEE 608 class project final report, 1998.

[15] C. Rolland, ON/OFF models to capture IP traffic structure.

[16] P. Bravo, Modelado fractal de tráfico de la red de Ingeniería Telemática, Tesis de grado Universidad Carlos III de Madrid, 2004.

[17] M. Alzate, "Introduccion al tráfico autosimilar redes de comunicaciones," Revista Ingeniería, vol. 6, nº. 2, pp. 6-17, Universidad Distrital Francisco José de Caldas, 2001.

[18] M. Kulikovs and E. Petersons, "Remarks Regarding Queuing Model and Packet Loss Probability for the Traffic with Self-Similar Characteristics," International Journal of Electrical and Computer Engineering. vol 3, no. 2, pp. 84-90. May. 2008.

[19] G. Bolch, S. Greiner, H. de Meer and K. S. Trivedi, Queueing Networks and Markov Chains. United States: John Wiley \& Sons, Inc., 2006.

[20] R. Clegg, "Markov-modulated on/off processes for long-range dependent internet traffic," Preprint submitted to Elsevier, 2007.

[21] P. Flandrin, "Wavelet Analysis and Synthesis of Fractional Brownian Motion," IEEE Transactions, Information Theory, vol. 38, no. 2. pp. 910-917, Mar. 1992.
[22] A. Tewfik and M. Kim, "Correlation structure of the discrete wavelet coefficients of Fractional Brownian Motion," IEEE Transactions on Information Theory, vol. 38, no. 2, pp. 904-909, Mar. 1992.

[23] H. Hsu, Theory and Problems of Probability, Random Variables, \& Random Processes, McGraw-Hill, 1997.

[24] I. Antonios and L. Lipsky, A Performance Model of User delay in On/Off Heavy-Tailed traffic. Com. Science \& Engineering, 2003.

[25] W. Willinger, M. Taqqu, R. Sherman and D. Wilson, "Self-Similarity Through High Variability: Statistical Analysis of Ethernet LAN Traffic at the Source Level," IEEE/ ACM Networking, vol. 5, no 1. Feb. 1997.

[26] C. Pesee, "Long-Range Dependence of Financial Time Series Data," International Journal of Human and Social Sciences, vol 4, no. 15, pp 1103-1107. 2009.

[27] B. Ray and R. Tsay, Identifying Common Long-range Dependence in a Vector Time Series, 1997.

[28] M. Alzate y F. Vega, "Predecibilidad del tráfico en redes modernas de telecomunicaciones," Revista Ingeniería, vol. 7, no. 2, pp. 21-30. Universidad Distrital Francisco José de Caldas, 2003.

[29] K. Park, QoS in Packet Networks. United States: Springer 2005.

[30] K. Chiang, M. Gurusamy, Y. Liu and M. Hoang, Quality of Service in Optical Burst Switched Networks. United States: Springer Science + Business Media, Inc. 2007. 
[31] W.E Leland, M.S. Taqqu, W. Willinger and D.V. Wilson, "On the self-similar nature of Ethernet traffic (extended version)," IEEE/ ACM Transactions on Networking, vol. 2, no. 1, pp. 1-15. Feb. 1994.

[32] P. Pruthi and A. Erramilli, "Heavy-tailed on/off source behavior and self-similar traffic," International Conferenceon Communications, Seattle, Jun. 1995.

[33] S. L. Cajamarca y C. Torres, Análisis y Modelado de Tráfico de datos con aplicación a redes de acceso ADSL. Pontificia Universidad Javeriana, Sep. 2002.

[34] T. D. Dang, B. Sonkoly and S. Molnar, Fractal Analysis and Modeling of VoIP Traffic, 2004.

[35] K. Kassev, Y. Mihov, B. Tsankov, "Performance Analysis of Call Admission Control for Streaming Traffic with Activity Detection Function," International Book Series. Information Science and Computing, no. 14, pp. 47-52, 2009.

[36] R. Vallejos, A. Zapata and M. Aravena, Fast blocking probability evaluation of end-toend optical burst switching networks with non-uniform ON-OFF input traffic model, vol. 13, no. 2, pp. 217-226. Apr. 2007.

[37] C. Chen, Y. Xu and L. Zhang, "Some Remarks on ON/OFF Network Traffic," Workshop on Power Electronics and Intelligent Transportation System, Guangzhou. 2008.

[38] B. P. Tsankov, P. H. Koleva, "ON-OFF-2ON Traffic Model for Packetized Voice over Wireless Both-Way Transmission Media," The Third International Conferenceon Digital Telecommunications, Bucharest, 2008.
[39] L. Aspirot, Regresión no paramétrica para datos funcionales no estacionarios, TesisMagistral, Universidad de la República. Montevideo, Uruguay, Sep. 2008.

[40] Q. Wang and T. Zhang, Source Traffic Modeling in Wireless Sensor Networks for Target Tracking, Oct. 2008.

[41] Y. Audzevich, L. Bodrog, Y. Ofek and M. Telek, Scalable Model for Packet Loss Analysis of Load-Balancing Switches with Identical Input Processes, 2009.

[42] C. Dombry and I. Kaj, The on-off network traffic model under intermediate scaling, Abr. 2010.

[43] R. Taylor, A. Micolich and David Jonas, "Fractal expressionism (A comparison of the paintings of Jackson Pollock to a Lévy flight model)," Physics World, vol. 12, no. 10 , Oct. 1999.

[44] S. Andreev, Y. Koucheryavy, A. Anisimov and A. Turlikov, Practical Traffic Generation Model for Wireless Networks. 2010 .

[45] C. Blondia, "A discrete-time batch Markovian arrival process as B-ISDN traffic model," Journal PerfomanceEvaluation, vol. 64, no. 1. Jan.1993.

[46] C. Eklund, R. Marks, S. Ponnuswamy, K. Stanwood and N. van Waes, WirelessMAN: Inside the IEEE 802.16 Standard for Wireless Metropolitan Area Networks, 1o ed. Wiley-IEEE Press, 2006.

[47] J. Vardakas, I. Moscholios, M. Logothetis and V. Stylianakis, "ON-OFF traffic models for a hybrid TDM-WDM PON with dynamic wavelength allocation". 7th International Symposium on Commu- 
nication Systems Networks and Digital Signal Processing (CSNDSP), Greece, Jul. 2010.

[48] G. Iacovoni\& S. Morsa, G. Iacovoni and S. Morsa, "Efficient simulation of a queueing system fed by general On/Off inputs," IEEE International Conference on Communications, Italy. 2005.

[49] S. Sarvotham, R. Riedi and Baraniuk, "Network and user driven alpha-beta onoff source model for network traffic," Ene. 2005.

[50] I. D. Moscholios, M. D. Logothetis\& G. K. Kokkinakis, "A multirate batched Poisson loss model of ON-OFF traffic sources: Call-level performance evaluation," Computer Networks, vol. 48, no 3, pp. 335-350. Jun. 2005.

[51] X. Yang, Designing Traffic Profiles for Bursty Internet Traffic. MIT Laboratory for Computer Science.

[52] I. Papapanagiotou, J.S. Vardakas, G.S. Paschos, M.D. Logothetis and S.A. "Kotsopoulos, Performance Evaluation of IEEE 802.11e based on ON-OFF Traffic Model, 2007.

[53] X. Tao, Traffic Balancing: A Method for Exploiting System Capacity in Wireless Ad Hoc Networks, Doctoral Thesis, Nov. 2005.

[54] M. Wang, Z.S. Wanga, Wei Lu, J.L. Lin and D.R. Chen, Performance of a joint CDMA/PRMA protocol with heavy-tailed ON/OFF source. 1999.
[55] D. K. Kim \& D. K. Sung, Capacity Estimation for an SIR-Based Power-Controlled CDMA System Supporting ON-OFF Traffic, vol. 5, no 3. May. 1999.

[56] T. Chen, Characterization of ATM On-Off Traffic from Cell Traffic Measurements, Jun. 1999.

[57] G. Niculescu and L. Ioan, Time-Saving Event Driven Simulation of ATM ON/OFF Traffic Sources. 2000.

[58] D. Zaragoza and C. Belo, Experimental validation of the ON-OFF packet-level model for IP traffic, Sep. 2006.

[59] H. Schwefel, "Behavior of TCP-like elastic traffic at a buffered bottleneck router," Twentieth Annual Joint Conference of the IEEE Computer and Communications Societies. Proceedings. IEEE. Anchorage. 2001.

[60] A. Wierman, T. Osogami, and J. Olsen, Modeling TCP-Vegas under On/Off Traffic, vol. 31, no. 2, Sept. 2003.

[61] H. P. Schwefela \& L. Lipsky, Impact of aggregated, self-similar ON/OFF traffic on delay in stationary queueing models. 2001 .

[62] M. Kulikovs and E. Petersons, Remarks Regarding Queuing Model and Packet Loss Probability for the Traffic with SelfSimilar Characteristics, May. 2008.

[63] I. D. Moscholios \& M. D. Logothetis, Call-Burst Blocking Probabilities of ONOFF Traffic Sources under the Bandwidth Reservation Policy. 2004. 